\title{
Caries prevention - little evidence for use of chlorhexidine varnishes and gels
}

\section{Abstracted from}

Walsh T, Oliveira-Neto JM, Moore D.

Chlorhexidine treatment for the prevention of dental caries in children and adolescents. Cochrane Database Syst Rev 2015; 4: CD008457. DOI: 10.1002/14651858.CD008457.pub2.

Address for correspondence: Luisa Fernandez Mauleffinch, Managing Editor, Cochrane Oral Health Group, School of Dentistry, The University of Manchester, JR Moore Building, Oxford Road, Manchester, M13 9PL, UK. E-mail: luisa.fernandez@manchester.ac.uk

\section{Question: Are chlorhexidine-containing oral products (toothpastes, mouthrinses, varnishes, gels, gums and sprays) effective in preventing caries in children and adolescents?}

Data sources Cochrane Oral Health Group Trials Register, Cochrane Central Register of Controlled Trials (CENTRAL), Medline, Embase and CINAHL.

Study selection Randomised controlled trials (RCTs) comparing the effects of chlorhexidine-containing oral products with no treatment or placebo on dental caries, and comparing the effects of one chlorhexidine-containing oral product with another were considered. Data extraction and synthesis Two reviewers independently extracted trial data and assessed risk of bias. Owing to the clinical diversity of the included studies with respect to age, composition of intervention, and variation in outcome measures and followup, meta-analysis was not carried out. A narrative synthesis was presented.

Results Eight trials were included. Six were at high risk of bias and two at unclear risk. Six trials compared chlorhexidine varnish with placebo or no treatment. Data from two trials (690 patients) one using $10 \%$ chlorhexidine varnish and the other, $40 \%$, showed that this led to an increase in the DMFS increment in the varnish group of 0.53 ( $95 \% \mathrm{Cl} ;-0.47$ to 1.53 ). Only one trial involving 496 patients and using $10 \%$ chlorhexidine varnish provided usable data for elevated mutans streptococci levels $>4$ with RR $0.93(95 \% \mathrm{Cl} ; 0.80$ to 1.07 ). Two trials (487 patients) compared $0.12 \%$ chlorhexidine gel with no treatment in the primary dentition. The presence of new caries gave rise to a $95 \%$ confidence interval that was compatible with either an increase or a decrease in caries incidence (RR 1.00, 95\% $\mathrm{Cl} 0.36$ to 2.77 ).

Conclusions The authors found little evidence from the eight trials on varnishes and gels included in this review to either support or refute the assertion that chlorhexidine is more effective than placebo or no treatment in the prevention of caries or the reduction of

This paper is based on a Cochrane Review published in the Cochrane Library 2015, issue 4 (see www.thecochranelibrary.com for information). Cochrane Reviews are regularly updated as new evidence emerges and in response to feedback, and the Cochrane Library should be consulted for the most recent version of the review. mutans streptococci levels in children and adolescents. There were no trials on other products containing chlorhexidine such as sprays, toothpastes, chewing gums or mouthrinses. Further high quality research is required, in particular evaluating the effects on both the primary and permanent dentition and using other chlorhexidinecontaining oral products.

\section{Commentary}

Dental caries is one of the commonest diseases in the world ${ }^{1}$ yet is almost completely preventable with a combination of oral hygiene using anticariogenic agents, typically the use of fluoride, and dietary measures. As acidogenic bacteria in dental plaque cause local demineralisation of the tooth that can lead to cavity formation there is the potential for antibacterial agents to contribute to caries reduction.

Chlorhexidine gluconate (a cationic bis-biguanide) has been used for many years as an antiplaque agent and has been shown to suppress Streptococcus mutans, so could be used for caries prevention. A number of chlorhexidine preparations are available, as toothpastes, gels, thymol-containing varnishes, chewing gums and sprays. This Cochrane review aimed to assess the effectiveness of these chlorhexidine-containing oral products on caries prevention in children and adolescents.

Only randomised controlled trials were considered for the review with the exclusion of split mouth RCTs as there is considerable potential for cross contamination. Standard Cochrane protocols for the conduct of the review were planned and followed where sufficient data were available.

The eight included trials were reported in 12 publications. Two of the trials were cluster design with randomisation at the school class level but these trials' authors did not take this clustering into account in their analysis. The review authors re-analysed these studies to take into account the clustering using a range of intraclass correlation coefficients.

A range of chlorhexidine preparations was used in the studies; $1 \%$, $10 \%, 40 \%$ chlorhexidine varnish, each with a different application regimen, and chlorhexidine gel with a concentration of $0.12 \%$. Most of the trials were considered to be at high risk of bias (six out of eight) and none at low risk of bias.

The review authors assessed the overall quality of evidence according to GRADE (Grading of Recommendations Assessment, Development and Evaluation) considering it to be of very low quality. 
No studies were found assessing chlorhexidine-based mouthrinses, toothpastes, chewing gums or sprays for the prevention of dental caries in children and adolescents and half of the included studies did not report adverse events. As there is a number of known adverse events that included staining of the teeth and tongue, mucosal soreness and desquamation, temporary taste disturbances, parotid gland swelling and hypersensitivity (including anaphylaxis) this is of concern.

At present there is little evidence that chlorhexidine could provide an alternative approach to caries prevention as there is good evidence for the use of topical fluorides in varnish, toothpastes, gels and mouthrinses ${ }^{2}$ as well as fissure sealants, ${ }^{3}$ so these approaches should be prioritised for caries prevention.
Derek Richards

Centre for Evidence-based Dentistry, Dental Health Services

Research Unit, University of Dundee, Dundee, Scotland, UK

1. Kassebaum NJ, Bernabé E, Dahiya M, Bhandari B, Murray C], Marcenes W. Global Burden of Untreated Caries: A Systematic Review and Metaregression. J Dent Res 2015; 94: 650-658.

2. Marinho VC, Higgins JP, Sheiham A, Logan S. One topical fluoride (toothpastes, or mouthrinses, or gels, or varnishes) versus another for preventing dental caries in children and adolescents. Cochrane Database Syst Rev 2004; 1:

[DOI: 10.1002/14651858.CD002780.pub2]

3. Ahovuo-Saloranta A, Forss $\mathrm{H}$, Walsh $\mathrm{T}$, et al. Sealants for preventing dental decay in the permanent teeth. Cochrane Database Syst Rev 2013; 3: [DOI: 10.1002/ 14651858.CD001830.pub4]

Evidence-Based Dentistry (2015) 16, 43-44. doi:10.1038/sj.ebd.6401091 